ISSN 0103-5150

Fisioter. Mov., Curitiba, v. 26, n. 3, p. 605-615, jul./set. 2013

Licenciado sob uma Licença Creative Commons

\title{
Influência de diferentes tipos de calçado na atividade eletromiográfica do músculo quadríceps de mulheres ao subir e descer degrau
}

\author{
Influence of different shoes on electromyographic \\ activity of asymptomatic and symptomatic women's \\ quadriceps muscles during up and down stairs
}

\author{
Valéria Mayaly Alves de Oliveira ${ }^{[a]}$, Laísla da Silva Paixão Batista ${ }^{[a]}$, Lucas Pereira Lopes de Souza ${ }^{[a]}$, \\ Ana Carolina Rodarti Pitangui ${ }^{[b]}$, Rodrigo Cappato de Araújo ${ }^{[c]}$ \\ [a] Graduanda do curso de Fisioterapia da Universidade de Pernambuco (UPE), Petrolina, PE - Brasil, e-mail: \\ valeria_mayaly@hotmail.com, mell-isa.net@hotmail.com,lpls@hotmail.com \\ [b] Doutora em Enfermagem em Saúde Pública pela Universidade de São Paulo (USP), professora adjunta do Departamento \\ de Fisioterapia da Universidade de Pernambuco (UPE), Petrolina, PE - Brasil, e-mail: carolinapitangui@yahoo.com.br \\ [c] Doutor em Engenharia Mecânica (Bioengenharia) pela Universidade Federal de Minas Gerais (UFMG), professor do \\ Programa Associado de Pós-Graduação em Educação Física da Universidade de Pernambuco (ESEF/UPE), Recife, PE - \\ Brasil, e-mail: rodrigocappato@yahoo.com.br
}

\section{Resumo}

Introdução: A síndrome da dor femoropatelar (SDFP) é caracterizada por dor difusa na região retropatelar, aumentada durante realização de tarefas diárias, tais como subir e descer escadas e ajoelhar. A SDFP tem maior prevalência em mulheres jovens e sua etiologia não é totalmente conhecida. Objetivo: Avaliar a influência do uso de diferentes tipos de calçados na atividade dos músculos estabilizadores da patela durante as tarefas de subir e descer degrau. Materiais e métodos: Participaram do estudo dez mulheres saudáveis e dez mulheres com SDFP. As voluntárias realizaram as tarefas de subir e descer um degrau em três situações: descalças, usando tênis e salto alto de $10 \mathrm{~cm}$. Durante a realização das tarefas, foi registrada com eletrodos superficiais a atividade eletromiográfica dos músculos vasto medial oblíquo (VMO), vasto lateral (VL) e reto femoral (RF). A comparação intergrupos foi realizada com o teste t não pareado; para a análise intragrupo, utilizou-se ANOVA com medidas repetidas e post hoc Tukey, todos com um nível de significância de 5\%. Resultados: Os resultados demonstraram, no grupo controle, aumento da atividade do VMO nas tarefas 
concêntrica e excêntrica realizadas com salto alto. No entanto, no grupo SDFP, a atividade do VMO não foi influenciada em nenhuma das condições testadas. Não foram observadas diferenças estatísticas na razão VMO/VL e no tempo de ativação dos músculos. Conclusão: Os resultados sugerem que em mulheres assintomáticas uso do calçado de salto alto modificou a atividade de todos os músculos. Entretanto, mulheres com SDFP não apresentaram alterações no VMO.

Palavras-chave: Síndrome da dor femoropatelar. Eletromiografia. Sapatos.

\begin{abstract}
Introduction: Patellofemoral pain syndrome (PPS) is characterized by diffuse pain in the retropatellar region, increased by daily tasks such as up and down stairs or kneeling. The PPS has a higher prevalence in young women and its etiology is not fully understood. The purpose of this study was to assess the influence of different types of shoes on the patella stabilizer muscles during the tasks up and down stairs. Materials and methods: Ten healthy control women and ten women with patellofemoral pain syndrome (PPS) participated in this study. The subjects performed up and down stair tasks under three conditions; barefoot, with tennis shoes and $10 \mathrm{~cm}$ high heel shoes. Electromyographic (EMG) activity was recorded from vastus medialis oblique (VMO), vastus lateralis (VL) and Rectus femoris (RF) muscles during the tasks using surface differential electrodes connected to an EMG system. The intergroup comparison was realized using the unpaired test $t$ and the intragroup using the repeated measures ANOVA post hoc Tukey, all with level of significance of 5\%. Results: Results demonstrated higher EMG activity for the VMO muscles during the eccentric and concentric phases performed with high heeled-shoes in the control group. However, in the PPS group, the VMO activity did not influence in any of the proposed conditions. There were no differences in VMO/VL ratio and onset timing for the both groups. Conclusions: The results suggest that in asymptomatic women using high-heeled shoes changed activity of all muscles. However, women with PFPS showed no changes in VMO.
\end{abstract}

Keywords: Patellofemoral pain syndrome. Electromyography. Shoes.

\title{
Introdução
}

A síndrome da dor femoropatelar (SDFP), de etiologia multifatorial, é uma desordem musculoesquelética que acomete principalmente mulheres jovens e sua queixa é frequentemente encontrada em clínicas de reabilitação (1-3). A SDFP é definida como uma dor na região anterior do joelho e/ou retropatelar, decorrente de alterações estruturais e biomecânicas da articulação femoropatelar (4-7).

Embora a etiologia da SDFP não esteja bem definida, acredita-se que o mau alinhamento e a instabilidade patelar são os principais fatores causais dessa desordem. Segundo alguns autores $(4,8,9)$, as alterações de estabilidade e alinhamento da patela podem ser causadas pelo desequilíbrio das estruturas estáticas, tais como retração do retináculo lateral, encurtamento do trato iliotibial, alterações do posicionamento patelar e aumento do ângulo Q. Essas alterações favorecem o desalinhamento da patela e o aumento da compressão mecânica na cartilagem articular e osso subcondral, o que explica o quadro álgico $(10,11)$.
Além disso, a instabilidade femoropatelar pode ser causada por alterações no antagonismo funcional das diferentes porções do músculo quadríceps, seja no aspecto da intensidade ou início de ativação do vasto medial oblíquo (VMO) e vasto lateral (VL), considerados os principais estabilizadores dinâmicos dessa articulação $(12,13)$. Alguns autores $(5,14-18)$ afirmam que o desequilíbrio de forças e o tempo de ativação entre VMO e VL podem causar o desalinhamento femoropatelar e o surgimento de quadro álgico, principalmente durante tarefas em que essa articulação é mais exigida, tais como agachar e subir ou descer escadas.

No entanto, alguns autores $(19,20)$ relataram não ter encontrado alterações na atividade e no tempo de ativação dos músculos estabilizadores em indivíduos com SDFP, sugerindo que o desequilíbrio entre VMO e VL não seria um fator determinante. Por fim, Dye (21) sugere que o desequilíbrio dos estabilizadores estáticos e dinâmicos, quando presente de forma isolada, não predispõe o desenvolvimento da SDFP, mas sim a associação de diversos fatores. 
Estudos recentes $(3,4,22)$ têm investigado a influência do uso de calçados de salto alto na biomecânica dos membros inferiores. Os resultados demonstram que o uso do salto alto altera a distribuição do peso no membro inferior, modifica a atividade dos músculos e proporciona um aumento da sobrecarga na articulação do joelho. 0 uso regular desse tipo de calçado contribui para o surgimento de alterações da mecânica das articulações do joelho e femoropatelar, podendo evoluir para processos articulares degenerativos e osteoartrite.

Partindo do pressuposto de que as mulheres são as mais acometidas tanto pela osteoartrite quanto pela SDFP e de que essa população faz uso de calçados com salto alto, supõe-se que exista relação entre o tipo de calçado e o comprometimento da articulação femoropatelar. No entanto, não foram encontrados na literatura estudos que avaliassem a influência de diferentes tipos de calçados na ativação dos músculos estabilizadores da patela em mulheres com e sem SDFP durante as tarefas de subir e descer um degrau.

Sendo assim, o objetivo do presente estudo foi avaliar a influência do tipo de calçado na atividade e no tempo de disparo eletromiográfico dos músculos estabilizadores da patela durante as tarefas de subir e descer um degrau, em mulheres com e sem SDFP.

\section{Materiais e métodos}

\section{Amostra}

Selecionaram-se 20 voluntárias adultas jovens e sedentárias, divididas em dois grupos: grupo controle, composto por dez voluntárias com média de idade de 21,3 $\pm 3,13$ anos, peso médio de 53,42 $\pm 3,65$ $\mathrm{kg}$ e estatura média de 1,60 $\pm 0,06 \mathrm{~m}$; e grupo SDFP, composto por dez voluntárias com média de idade de $20,20 \pm 3,46$ anos, peso médio de 53,14 $\pm 5,25 \mathrm{~kg}$ e estatura média de 1,60 $\pm 0,04 \mathrm{~m}$. Todas as voluntárias submetidas ao estudo assinaram previamente o Termo de Consentimento Livre e Esclarecido, e o trabalho foi aprovado pelo Comitê de Ética da Universidade de Pernambuco (UPE), sob o protocolo n. 202/09.

Os critérios gerais de inclusão para o estudo foram: idade entre 18 e 30 anos, sedentárias por pelo menos três meses, que calçassem sapatos de número 36 e que fizessem uso de calçados de salto alto por pelo menos três vezes por semana. Para alocação no grupo controle, as mulheres não poderiam apresentar história de dor, cirurgia, trauma ou lesão no sistema osteomioarticular no membro inferior; assinalar dor zero na escala EVA (Escala Visual Analógica) em relação à articulação femoropatelar na semana anterior à avaliação. Para alocação no grupo SDFP, as voluntárias deveriam apresentar dor prévia na região anterior ou retropatelar no mínimo em três das seguintes atividades: sentar prolongado, correr, subir ou descer escadas, agachar, ajoelhar e na contração isométrica do quadríceps. Ainda não poderiam ter histórico cirúrgico no membro inferior; apresentar no mínimo um sinal e sintoma clínico observados na avaliação funcional (entre eles: aumento do ângulo $Q$ sinal de baioneta, patela medializada, pronação subtalar excessiva, sensibilidade à palpação das facetas patelares, torção tibial externa); assinalar no mínimo dois na EVA relativa à articulação femoropatelar na semana anterior à avaliação; não apresentar dor nos últimos dois meses.

Para ambos os grupos foram excluídas as voluntárias que relataram uso de medicamentos e/ou foram submetidas a fisioterapia prévia durante o período de seis meses anteriores e distúrbios neurológicos e/ou neuromusculares.

\section{Eletromiografia de superfície}

Para aquisição dos registros EMG foram utilizados três canais do sistema Myosystem Br-1 da Datahominis Tecnologia Ltda (Uberlândia, Brasil). 0 equipamento possuía aterramento e aquisição simultânea comum para os canais, filtro de banda $10 \mathrm{~Hz}$ a $5 \mathrm{KHz}$; três estágios de amplificação, impedância dos canais de $10 \mathrm{G} \Omega$ em modo diferencial, 24 bits e faixa resolução dinâmica, faixa de amplitude de $-10 \mathrm{~V}$ a $+10 \mathrm{~V}$ e placa conversora analógica-digital. Para visualização e processamento dos sinais, foi utilizado o programa Myosystem Br-1, versão 3.5.

Os sinais mioelétricos foram captados por meio de eletrodos ativos de superfícies simples diferenciais de ganho de 20 vezes, compostos por duas barras retangulares paralelas de prata pura (Ag), da Datahominis Tecnologia Ltda (Uberlândia, Brasil). Para redução de ruído e aquisição, empregou-se um eletrodo circular com tamanho de $3 \mathrm{~cm}^{2}$ como eletrodo de referência, localizado na porção distal da tíbia.

O sinal EMG bruto foi utilizado para derivar os valores de amplitude eletromiográfica obtidos no cálculo do Root Mean Square (RMS). 0 início da atividade do VMO e VL foi definido como o primeiro intervalo de $25 \mathrm{~ms}$, no qual o nível de ativação foi maior do que dois desvios padrões do valor coletado durante o repouso. 
Definidos os tempos de início da atividade do VMO e do VL, calculou-se a média aritmética entre as subtrações dos valores do VMO pelos valores do VL de todos os indivíduos ( $\Delta \mathrm{T}$-s). Os dados foram coletados a $4.000 \mathrm{Hze}$ filtros digitais passa-baixo $500 \mathrm{~Hz}$ e passa-alto de $15 \mathrm{~Hz}$ foram aplicados. Os valores de RMS foram normalizados pelo valor médio da amplitude eletromiográfica obtido em três contrações isométricas voluntárias máximas (CIVM) da musculatura extensora. Além disso, procedeu-se à análise da proporção de ativação dos músculos VMO e VL, definida pela razão (VMO/VL) dos valores de amplitude do sinal EMG.

\section{Procedimentos}

Inicialmente, realizaram-se avaliação física para coleta de dados antropométricos e testes clínicos para confirmação do grupo de alocação de cada voluntária. Após a avaliação, foi sorteada a ordem das tarefas e realizadas tricotomia e limpeza local com álcool para redução da impedância da pele. Posteriormente, os eletrodos de superfície foram fixados com esparadrapo nos músculos reto femoral (RF), vasto lateral (VL) e vasto medial oblíquo (VMO), de forma a respeitar o direcionamento das fibras. 0 músculo $\mathrm{RF}$ foi posicionado em $50 \%$ da linha entre a espinha ilíaca superior e a porção superior da patela de acordo com o SENIAM (do inglês, Surface EMG for Non-Invasive Assessment of Muscles, ou Eletromiografia de superfície para avaliação não invasiva de músculos) (23). Entretanto, para fixação dos eletrodos nos músculos VMO e VL, foram seguidos os procedimentos descritos por Grossi et al. (2). Para o músculo VMO, o eletrodo foi posicionado no ventre muscular com uma inclinação de $55^{\circ}$ enquanto o VL foi posicionado a $10 \mathrm{~cm}$ da borda superolateral da patela, contendo uma inclinação de aproximadamente $17^{\circ}$. 0 membro dominante foi o critério de escolha da musculatura avaliada pelo grupo controle, ao passo que no grupo com SDFP, a avaliação EMG foi realizada pelo membro acometido ou com maior acometimento no caso de SDFP bilateral.

Após a colocação dos eletrodos, foram realizadas três contrações isométricas voluntárias máximas (CIVM) da musculatura extensora da perna, com as voluntárias sentadas em uma cadeira extensora com o suporte de membros inferiores travado, mantendo $90^{\circ}$ de flexão de quadril e joelho. As voluntárias foram orientadas a realizar três CIVM mantidas por 4 segundos contra o suporte, com intervalos de 2 minutos entre cada contração. Finalizada a última CIVM, foi respeitado um período de 10 minutos de repouso para o início das duas tarefas:

a) Subir degrau (contração concêntrica)

As voluntárias foram posicionadas em frente a uma escada de metal com dois degraus, com $20 \mathrm{~cm}$ de altura cada. Foi solicitado que as voluntárias posicionassem o membro inferior dominante sobre o primeiro degrau, mantendo os pés alinhados e a uma distância equivalente à largura dos ombros. Após comando verbal e com auxilio visual e sonoro de um metrônomo, as voluntárias foram orientadas a subir o degrau da escada em um tempo de 1,20 segundos (Figura 1A).

\section{b) Descer degrau (contração excêntrica)}

As voluntárias foram posicionadas sobre o primeiro degrau da escada, com os pés em paralelo e a uma distância equivalente à largura dos ombros. Após comando verbal, foram orientadas a descer do degrau com o membro não dominante, o que exigiria uma contração excêntrica do músculo quadríceps do membro contralateral. Essa tarefa, assim como a anterior, foi realizada em 1,20 segundos também com auxílio de um metrônomo (Figura 1B).

Ambas as tarefas foram realizadas com as voluntárias em três condições: descalças; utilizando tênis; usando sapato com salto de $10 \mathrm{~cm}$. Em cada condição, as voluntaria realizaram o movimento por três vezes, com intervalo de 2 min. entre as repetições. Para cada troca de calçado foi concedido às voluntárias um período de 10 min. para adaptação ao calçado; para tanto, as participantes ficavam de pé e davam alguns passos. A ordem para a execução das tarefas e dos tipos de calçados foi realizada de forma aleatória. Além disso, antes dos registros das tarefas foi permitido que as voluntárias fizessem três testes de cada tarefa utilizando o metrônomo para treinamento e aprendizagem da velocidade do movimento.

\section{Análise estatística}

Todos os testes estatísticos foram realizados no programa SPSS, versão 16.0. Inicialmente, o teste Shapiro-Wilk foi empregado para a confirmação de que as variáveis estudadas apresentavam distribuição 


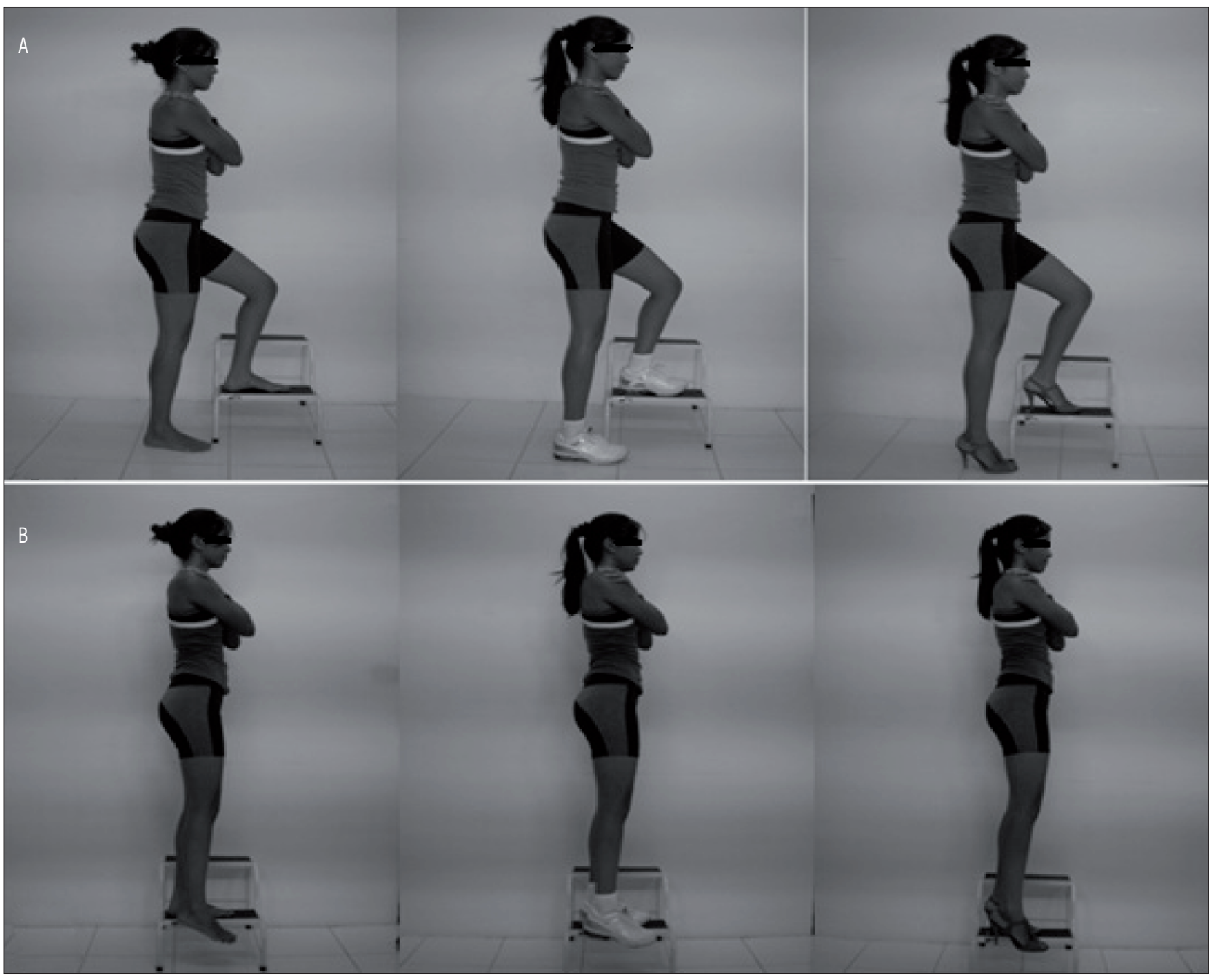

Figura 1 - Posição de execução das atividades de subir o degrau (A) e descer o degrau (B) com a voluntária descalça, com tênis e salto alto

Fonte: Dados da pesquisa.

normal. Para análise da influência dos diferentes tipos de calçado na atividade eletromiográfica dos músculos estudados, foi aplicado o teste ANOVA com medidas repetidas e post hoc Tukey. Já para as comparações intergrupos, foi utilizado o teste $t$ não pareado. Em todos os testes estatísticos aplicados foi considerado um nível de significância de 5 \%.

\section{Resultados}

\section{Subir degrau}

Os resultados para o grupo controle indicaram que a atividade eletromiográfica dos músculos VMO, VL e RF foi estatisticamente maior quando a tarefa foi realizada com salto alto $(p<0,02)$. No entanto, nenhuma diferença significativa foi encontrada na comparação entre a realização da tarefa realizada com os pés descalços e com tênis $(\mathrm{p}>0,58)$. Além disso, em todas as situações os músculos VMO e VL não apresentaram diferença estatística em suas atividades ( $p>0,28)$, porém ambos apresentaram maior atividade EMG quando comparados ao músculo RF $(\mathrm{p}<0,03)$ (Tabela 1).

Em relação ao grupo SDFP, foi observado que o uso do salto alto aumentou significantemente a atividade dos músculos VL e RF na comparação com as demais situações $(p<0,02)$. No entanto, não houve diferença nos valores de amplitude EMG do músculo VMO ( $p>0,09$ ). A atividade EMG dos músculos VMO e VL foi estatisticamente maior do que a atividade do RF 
Tabela 1 - Atividade EMG normalizada (\% RMS) dos músculos vasto medial oblíquo, vasto lateral e reto femoral durante a tarefa de subir escada utilizando tênis, salto e estando descalço para o grupo controle e SDFP

\begin{tabular}{|c|c|c|c|c|c|c|}
\hline \multirow[b]{3}{*}{ VMO* } & \multicolumn{3}{|c|}{ Controle } & \multicolumn{3}{|c|}{ SDFP } \\
\hline & SED & SET & SES & SED & SET & SES \\
\hline & $14,5 \pm 5,1$ & $14,4 \pm 5,3$ & $20,9 \pm 5,3^{a, b}$ & $16,2 \pm 10,4$ & $16,1 \pm 10,2$ & $15,5 \pm 11,4$ \\
\hline VL* & $13,2 \pm 5,5$ & $13,1 \pm 6,1$ & $16,9 \pm 6,2 \mathrm{a}, \mathrm{b}$ & $14,5 \pm 10,2$ & $15,2 \pm 12,0$ & $20,7 \pm 11,1^{a, b}$ \\
\hline $\mathrm{RF}^{*}$ & $5,1 \pm 2,4$ & $5,4 \pm 2,1$ & $8,2 \pm 3,4^{a, b}$ & $6,2 \pm 4,3$ & $7,6 \pm 5,0$ & $11,8 \pm 6,0 \mathrm{ab}$ \\
\hline
\end{tabular}

Legenda: $\mathrm{VMO}=$ músculo vasto medial oblíquo; $\mathrm{VL}=$ músculo vasto lateral; $\mathrm{RF}=$ músculo reto femoral; $\mathrm{SET}=$ subir escada utilizando tênis; SES = subir escada utilizando salto; SED = subir escada descalço; SDFP = síndrome da dor femoropatelar; * $=p<0,05$; $a=$ representa diferença significativa entre SED e SES; $b$ = diferença significativa entre SET e SES.

Fonte: Dados da pesquisa.

Nota: Médias e desvios padrões da atividade EMG normalizada apresentada em RMS, expressos como percentagem da CIVM (\%RMS).

quando as voluntárias realizaram a tarefa descalças ( $\mathrm{p}<0,02$ ) e utilizando o tênis ( $\mathrm{p}<0,03)$; mas quando utilizaram o salto alto, não foi observado diferença na atividade EMG entre os músculos ( $p>0,28)$.

\section{Descer degrau}

Para ambos os grupos, foi observado que em todas as situações os músculos VMO e VL apresentaram maior atividade EMG que o músculo $\mathrm{RF}$ ( $\mathrm{p}<0,01$ ). Além disso, em ambos os grupos, o músculo VL apresentou maior atividade nas tarefas realizadas com sapato de salto alto em relação as demais situações $(\mathrm{p}<0,02)$, e o músculo RF apresentou maior atividade com salto somente quando comparada com a realização da tarefa com as voluntárias descalças ( $\mathrm{p}=$ 0,03 ). A atividade EMG do VMO somente se apresentou aumentada durante a realização da tarefa com salto alto nas voluntárias do grupo controle $(\mathrm{p}<0,01)$. No grupo SDFP não foi observada alteração significativa da atividade EMG do VMO quando utilizado o sapato com salto alto $(p>0,11)$. Nenhuma diferença foi encontrada na comparação entre descalço e tênis ( $p>0,46)$ (Tabela 2).

\section{Razão VMO/VL e início da ativação}

Em ambos os grupos, não foram observadas diferenças estatísticas nos valores da razão VMO/VL

Tabela 2 - Atividade EMG normalizada (\% RMS) dos músculos vasto medial oblíquo, vasto lateral e reto femoral durante a tarefa de subir escada utilizando tênis, salto e estando descalço para o grupo controle e SDFP

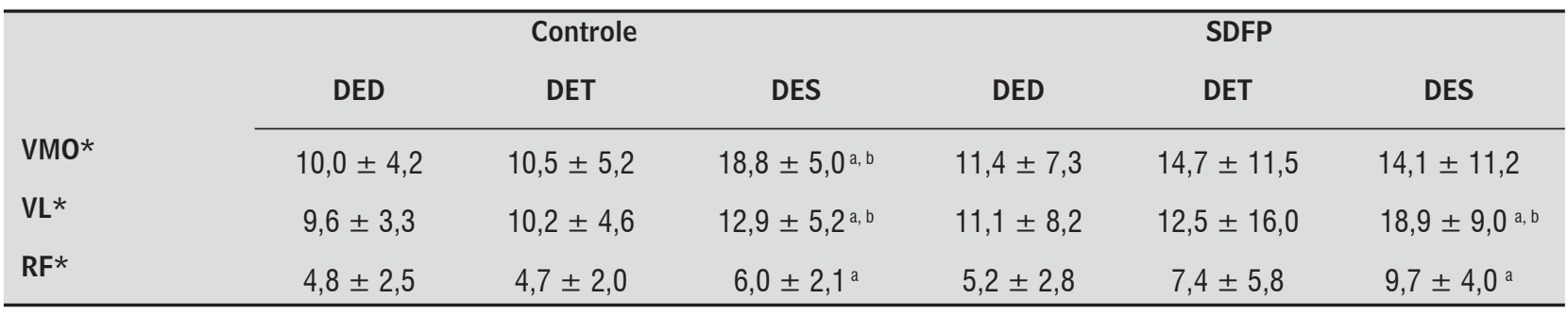

Legenda: VMO = músculo vasto medial oblíquo; $\mathrm{VL}=$ músculo vasto lateral; $\mathrm{RF}=$ músculo reto femoral; $\mathrm{DET}=$ descer escada utilizando tênis; DES = descer escada utilizando salto; DED = descer escada descalço; SDFP = síndrome da dor femoropatelar; * $=p<$ 0,$05 ; a$ = diferença significativa entre DED e DES; $b=$ diferença significativa entre DET e DES.

Fonte: Dados da pesquisa.

Nota: Médias e desvios padrões da atividade EMG normalizada apresentada em RMS, expressa como percentagem da CIVM (\%RMS). 
na comparação entre a realização das tarefas com diferentes tipos de calçados ( $\mathrm{p}>0,12$ ) (Gráfico 1). Isso confirma que tanto o tipo de calçado utilizado, quanto o tipo de contração muscular, seja ela concêntrica e excêntrica, não foram capazes de influenciar a razão VMO/VL.
Assim como observado na razão VMO/VL, a análise do tempo de disparo eletromiográfico dos músculos VMO e VL não demonstrou diferença estatística em nenhuma das comparações realizadas ( $p>0,26)$ (Tabela 3).

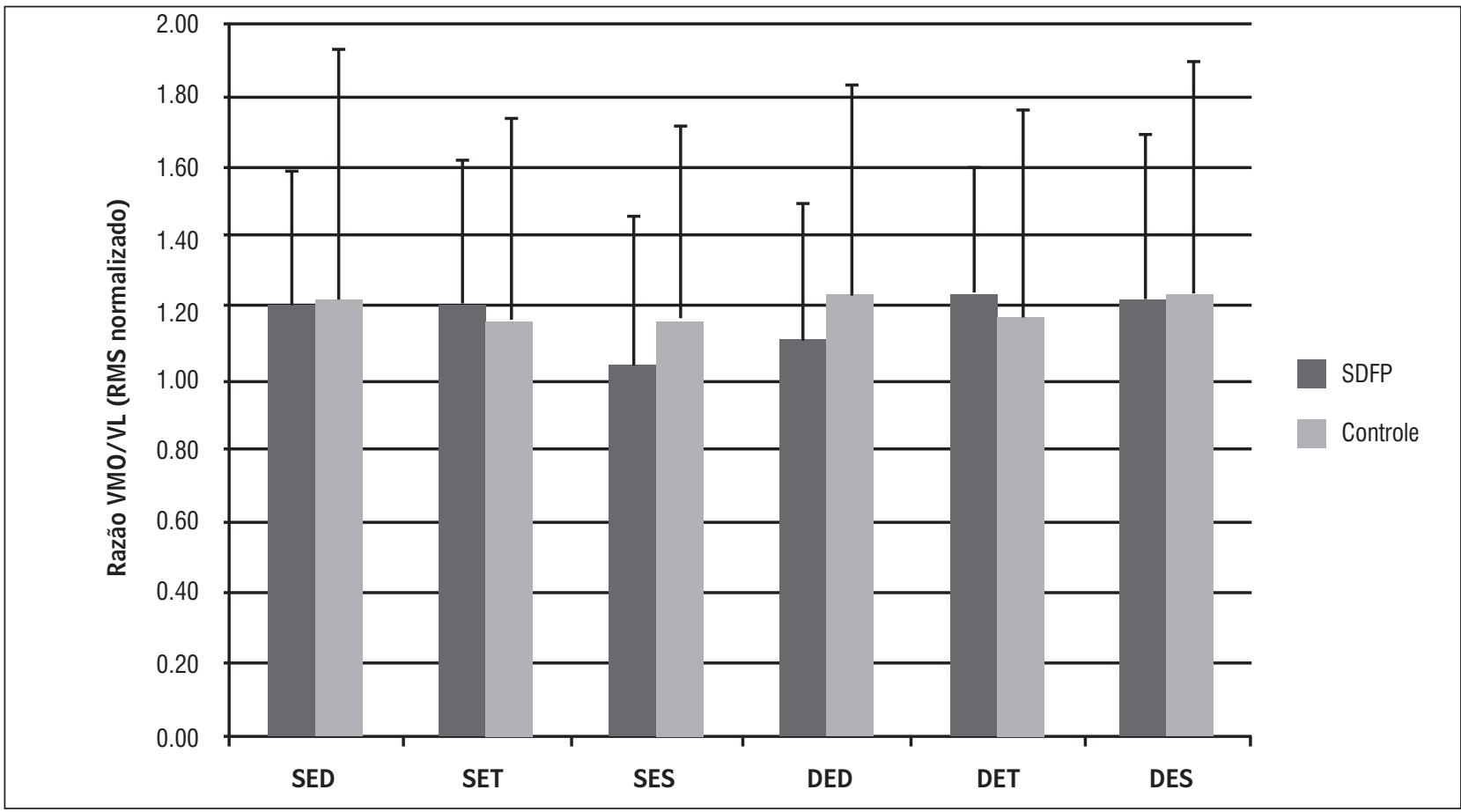

Gráfico 1 - Razão entre os músculos vasto medial oblíquo (VMO) e vasto lateral (VL) para os grupos controle e SDFP durante as atividades de subir e descer escada

Legenda: SED = subir escada descalço; SET = subir escada com tênis; SES = subir escada com salto; DED = descer escada descalço; $\mathrm{DET}=$ descer escada com tênis; DES = descer escada com salto.)Fonte: Dados da pesquisa.

Fonte: Dados da pesquisa.

Tabela 3 - Diferenças VMO-VL ( $\Delta \mathrm{t}-\mathrm{s})$ durante as atividades de subir e descer escada

\begin{tabular}{llll}
\hline & & Controle & SDFP \\
\hline \multirow{3}{*}{ Descer escada } & DED & $-0,07 \pm 0,10$ & $-0,05 \pm 0,09$ \\
& DES & $0,05 \pm 0,07$ & $0,02 \pm 0,02$ \\
& DET & $0,03 \pm 0,08$ & $0,03 \pm 0,10$ \\
\hline \multirow{3}{*}{ Subir escada } & SED & $-0,01 \pm 0,04$ & $-0,03 \pm 0,06$ \\
& SES & $-0,01 \pm 0,04$ & $-0,01 \pm 0,02$ \\
& SET & $-0,01 \pm 0,03$ & $-0,02 \pm 0,02$ \\
\hline
\end{tabular}

Legenda: SED = subir escada descalço; SET = subir escada com tênis; SES = subir escada com salto; DED = descer escada descalço; $\mathrm{DET}=$ descer escada com tênis; $\mathrm{DES}=$ descer escada com salto.

Fonte: Dados da pesquisa.

Notas: Médias e desvios padrões dos valores de início de ativação do VMO menos os valores de VL, expressos em segundos $(\Delta T-s)$; valores negativos indicam início prévio do VMO; nenhuma diferença significativa foi identificada nas comparações intra e intergrupos. 


\section{Discussão}

0 presente estudo buscou verificar as repercussões do uso de diferentes tipos de calçados sobre a atividade dos músculos estabilizadores da patela. Os resultados encontrados demonstram que durante as duas tarefas realizadas, a atividade eletromiográfica dos músculos VL e RF apresentam-se aumentadas quando a altura do calçado maior e que isso ocorre tanto em ambos os grupos, no sintomático e no assintomático. No entanto, no grupo SDFP a atividade eletromiográfica do músculo VMO não aumentou com o uso do salto alto, divergindo do grupo assintomático.

$\mathrm{O}$ aumento da amplitude eletromiográfica dos músculos avaliados e a ativação sincrônica do VMO e VL parecem compor um mecanismo de resposta que evita o aumento do ângulo de congruência patelar durante atividades dinâmicas (4), evitando a compressão excessiva entre as facetas articulares da patela contra os côndilos femorais (15). Atividade mais intensa do quadríceps ocorre quando o momento flexor do joelho aumenta devido à inclinação da articulação do tornozelo proporcionada pelo uso do salto alto $(3,24-26)$. Isso ficou confirmado no presente estudo, com o aumento da atividade eletromiográfica de todos os músculos quando realizadas as tarefas com o calçado de salto alto.

Além disso, alguns autores $(4,27,28)$ relatam que o uso do calçado de salto alto proporciona um aumento do momento externo adutor no joelho. Nesse sentido, a contração do músculo quadríceps cria um momento interno abdutor, que o objetivo de contrapor e equilibrar essas forças. Diante disso, o VMO deve também aumentar sua atividade para manter o alinhamento patelar adequado.

Somente um estudo (23) que avaliou a influência do uso de salto alto na atividade dos músculos estabilizadores da patela durante a atividade de subir uma escada observou, em mulheres assintomáticas, que esse tipo de calçado proporciona um aumento da intensidade de contração tanto do VMO quanto do VL. Contudo, a razão entre eles não aumenta, corroborando, assim, com nossos resultados.

No entanto, no grupo SDFP foi observado que a atividade do VMO não aumentou com o uso do salto alto, sugerindo que nessas voluntárias pode ter ocorrido uma resposta insuficiente do VMO. Segundo alguns autores $(29,30)$, dor e inflamação na articulação do joelho são responsáveis pela inibição neuromuscular do quadríceps, sendo o vasto medial o músculo mais frequentemente afetado. Portanto, esse fato pode explicar o resultado encontrado no presente estudo.

Edwards et al. (3), em estudo prévio, demonstraram que o aumento do salto foi responsável por uma maior atividade do músculo VL; quando voluntárias assintomáticas realizaram o movimento de levantar de um banco. Nossos achados divergem desses resultados, pois não foram observadas diferenças entre as atividades do VMO e VL; esse fato pode ser justificado pela diferença nas alturas dos saltos, visto que no presente estudo foi utilizado um salto de $10 \mathrm{~cm}$, enquanto no estudo anterior foi utilizado um salto de apenas $5 \mathrm{~cm}$. Outra diferença deve ser indicada: enquanto Edwards et al. (3) utilizaram em seu estudo um dispositivo de madeira para simulação do salto alto, em nosso estudo utilizou-se calçado de salto fino, que apresenta menor área de contato com o solo e por isso poderia influenciar a atividade dos músculos do membro inferior e tronco pela necessidade de manutenção da estabilidade articular e equilíbrio dinâmico. Essas diferenças podem ter influenciado não só o posicionamento, mas também a mecânica dos pés e membros inferiores.

Outra possível explicação para essa divergência está relacionada às diferenças biomecânicas existentes entre as tarefas executadas em cada estudo, considerando que Edwards et al. (3) analisaram tarefas bipodais, ao passo que no presente estudo foram executadas tarefas unipodais. Para a realização da tarefa unipodal de subir ou descer um degrau é necessária maior estabilização da pelve e do tronco, por meio do equilíbrio de forças entre músculos abdutores e adutores do quadril.

Segundo alguns autores (31-33), ao se realizar uma atividade com apoio unipodal, os músculos abdutores do quadril contraem-se a fim de manter o alinhamento da pelve. Entretanto, alterações no equilíbrio das forças dos músculos do quadril podem colaborar para o aumento do valgo dinâmico do joelho durante a realização de tarefas funcionais que envolvem descarga de peso sobre o membro inferior. Nesse sentido, essas alterações podem influenciar diretamente o alinhamento patelar (32).

De acordo com Coqueiro et al. (34), a influência dos músculos adutores do quadril no equilíbrio entre os estabilizadores patelares pode ser explicada pela forte relação anatômica entre os músculos adutores e o VMO. Segundo Hanten e Schulties (35), uma força originada do VMO quando se tem fraqueza dos adutores do quadril serviria apenas para puxar os tendões 
dos adutores em direção à patela; por outro lado, adutores do quadril fortalecidos dão ao VMO maior estabilidade para contrair. Assim, possivelmente a contração dos adutores durante essa atividade foi responsável por influenciar a ativação do VMO nos dois grupos, contribuindo para a identificação de diferença intergrupos. Entretanto, para confirmar esse resultado podem ser realizadas avaliações da atividade EMG dos músculos do quadril e avaliações cinemáticas e cinéticas.

Em relação ao tempo de ativação, Cavazzuti et al. (19) não identificaram diferença na comparação entre VMO e VL em indivíduos sintomáticos e assintomáticos durante tarefa em cadeia cinética fechada com apoio bipodal, rejeitando assim a hipótese de que os indivíduos portadores da SDFP apresentavam um retardo no tempo de ativação do VMO quando comparados a indivíduos normais. Da mesma forma, os resultados do presente estudo mostram que durante a realização de tarefas com apoio unipodal não há essa diferença, independentemente do tipo de calçado, rejeitando também essa hipótese.

0 presente estudo apresenta resultados preliminares e originais quanto à intensidade e tempo de ativação dos músculos estabilizadores patelares durante a corriqueira tarefa de subir e descer um degrau. No entanto, esse estudo apresenta algumas limitações, tais como amostra pequena, falta da análise cinética e cinemática do movimento. Portanto, estudos futuros devem avaliar tais aspectos, juntamente com o comportamento de mais músculos do membro inferior, principalmente, adutores e abdutores do quadril, em um grupo maior de mulheres.

\section{Conclusão}

Os resultados do presente estudo demonstraram que o uso do salto alto promoveu a alteração da atividade dos músculos RF, VMO e VL, em mulheres assintomáticas. No entanto, as mulheres com SDFP não apresentaram alterações na atividade do músculo VMO ao utilizarem salto alto. Esses achados sugerem que mulheres assintomáticas e com SDFP podem apresentar diferentes respostas ao uso do calçado com salto. No entanto, mais estudos são necessários para determinar se o uso regular do calçado com salto alto pode ser um fator que predispõe o desenvolvimento da SDFP.

\section{Referências}

1. Nakagawa TH, Muniz TB, Baldon RB, Serrão FV. Hip muscle's functional approach in the of patellofemoral pain syndrome treatment. Fisioter Mov. 2008;21(1):65-72.

2. Grossi DB, Felicio LR, Simões R, Coqueiro KRR, Monteiro-Pedro V. Electromyographic activity evaluation of the patella muscles during squat isometric exercise in individuals with patellofemoral pain syndrome. Rev Bras Med Esporte. 2005;11(3):159-63.

3. Edwards L, Dixon J, Kent JR, Hodgson D, Whittaker $\mathrm{V}$. Effect of shoe heel height on vastus medialis and vastus lateralis electromyographic activity during sit to stand. J Orthop Surg Res. 2008;3:2.

4. Cowan SM, Hodges PW, Bennell KL, Crossley KM. Altered vastii recruitment when people with patellofemoral pain syndrome complete a postural task. Arch Phys Med Rehabil. 2002;83(7):989-95.

5. Cabral CMN, Melin AMO, Sacco ICN, Marques AP. Physical therapy in patellofemoral syndrome patients: comparison of open and closed kinetic chain exercises. Acta Ortop Bras. 2008;16(3):180-5.

6. Fagan V, Delahunt E. Patellofemoral pain syndrome: a review on the associated neuromuscular deficits and current treatment options. Br J Sports Med. 2008;42(10):789-95.

7. Pulzatto F, Say KG, Siqueira AC, Santos GM, Grossi DB, Oliveira AS, et al. Step height influence on backward step-up exercise: an electromyographic study in healthy individuals and in those with patellofemoral pain syndrome. Acta Ortop Bras. 2005;13(4):168-70.

8. Cerny K. Vastus medialis oblique vastus lateralis muscle activity rations for selected exercises in persons with and without patellofemoral pain syndrome. Phys Ther. 1995;75(8):672-83.

9. Vaatainen U, Airaksinen O, Jaroma H, Kiviranta I. Decreased torque and electromyographic activity in the extensor thigh muscles in chondromalacia patellae. Int J Sports Med. 1995;16(1):45-59.

10. Besier TF, Draper CE, Gold GE, Beaupré GS, Delp SL. Patellofemoral joint contact area increases with knee flexion and weight-bearing. J Ortho Res. 2005;23(2):345-50. 
11. Grabiner MD, Koh TJ, Draganich LF. Neuromechanics of the patellofemoral joint. Med Sci Sports Exerc. 1994;26(1):10-21.

12. Lam PL, Ng GYF. Activation of the quadriceps muscle during semisquatting with different hip and knee positions in patients with anterior knee pain. Am J Phys Med Rehabil. 2001;80(11):804-8.

13. Grossi DB, Pedro VM, Bérzin F. Functional analysis of the patellar stabilizers. Acta Ortop Bras. 2004;12(2):99-104.

14. Grossi DB, Felício LR, Silvério GW. On set of electrical activity of patellar stabilizer muscles in subjects with patellofemoral pain. Acta Ortop Bras. 2009;17(5):297-9.

15. Grossi DB, Souza AC, Foerster B, Tannus A, Pereira JC, Terruggi JR, et al. Síndrome da dor femoropatelar eletromiografia, isocinética e ressonância magnética. Fisioter Bras. 2004;5(6):490-5.

16. Ribeiro AC, Grossi DB, Foerster B, Candolo C, Pedro VM. Electromyographic and magnetic resonance imaging evaluations of individuals with patellofemoral pain syndrome. Rev Bras Fisioter. 2010;14(3):221-8.

17. Cowan SM, Bennell KL, Hodges PW, Crossley KM, McConnell J. Delayed onset of electromyographic activity of vastus medialis obliquus relative to vastus lateralis in subjects with patellofemoral pain syndrome. Arch Phys Med Rehabil. 2001;82(2):183-9.

18. Cowan SM, Bennell KL, Crossley KM, Hodges PW, McConnell J. Physical therapy alters recruitment of the vasti in patellofemoral pain syndrome. Med Sci Sports Exerc. 2002;34(12):1879-85.

19. Cavazzuti L, Merlo A, Orlandi F, Campanini I. Delayed onset of electromyographic activity of vastus medialis obliquus relative vastus lateralis in subjects with patellofemoral pain syndrome. Gait Posture. 2010;32(3):290-5.

20. Souza DR, Gross MT. Comparison of vastus medialis obliquus: vastus lateralis muscle integrated electromyographic ratios between healthy subjects and patients with patellofemoral pain. Phys Ther. 1991;71(4):310-6.

21. Dye SF. Patellofemoral pain current concepts: an overview. Sports Med Arthrosc. 2001;9:264-72.

22. Santos CL, Noronha DO, Gomes CA, Fernandes PR, Fernandes J Filho. Repercussões biomecânicas do uso de salto alto na cinemática da marcha: um estudo retrospectivo de 1990 a 2007. Rev Ed Fís. 2008;143:47-53.
23. Hermens HJ, Freriks B, Merletti R, Stegeman D, Blok J, Rau G, et al. European recommendations for surface electromyography. Enschede: Roessingh Research and Development; 1999.

24. Yoon JY, An DH, Yoo WG, Kwon YR. Differences in activities of the lower extremity muscles with and without heel contact during stair ascent by young women wearing high-heeled shoes. J Orthop Sci. 2009;14(4):418-22.

25. Kerrigan DC, Johansson JL, Bryany MG, Boxer JA, Croce UD, Riley PO. Moderate-heeled shoes and knee joint torques relevant to the development and progression of knee osteoarthritis. Arch Phys Med Rehabil. 2005;86(5):871-5.

26. Opila-Correia KA. Kinematics of high-heeled gait. Arch Phys Med Rehabil. 1990;71(5):304-9.

27. Baliunas AJ, Hurwitz DE, Ryals AB, Karrar A, Case JP, Block JA, et al. Increased knee joint loads during walking are present in subjects with knee osteoarthritis. Osteoarthritis Cartilage. 2002;10(7):573-9.

28. Hertel J, Sloss BR, Earl JE. Effect of foot orthotics on quadriceps and gluteus medius electromyographic activity during selected exercises. Arch Phys Med Rehabil. 2005;86(1):26-30.

29. Fonseca ST, Cruz ABC, Lima SS, Seixas AFAM. Análise eletromiográfica dos músculos vasto medial oblíquo e vasto lateral em exercícios usados no tratamento da síndrome da dor patelofemoral. Rev Fisiot Univers SP. 2001;8(1):1-10.

30. Torry MR, Decker MJ, Viola RW, O'Connor DD, Steadman JR. Intra-articular knee joint effusion induces quadriceps avoidance gait patterns. Clin Biomech. 2000;15(3):147-59.

31. Nakagawa TH, Muniz TB, Baldon RDM, Maciel CD, Reiff RBM, Serrao FV. The effect of additional strengthening of hip abductor and lateral rotator muscles in patellofemoral pain syndrome: a randomized controlled pilot study. Clin Rehabil. 2008;22(12):1051-60.

32. Nakagawa TH, Moriya ETU, Dias C, Maciel CD, Serrao FV. Frontal plane biomechanics in males and females with and without patellofemoral pain. Med Sci Sports Exerc. 2012;44(9):1747-55.

33. Powers CM. The influence of altered lower-extremity kinematics on patellofemoral joint dysfunction: A theoretical perspective. J Orthop Sports Phys Ther. 2003;33(11):639-46. 
34. Coqueiro KR, Grossi DB, Bérzin F, Soares AB, Candolo $\mathrm{C}$, Pedro VM. Analysis on the activation of the VMO and VLL muscles during semisquat exercises with and without hip adduction in individuals with patellofemoral pain syndrome. J Electromyogr Kinesiol. 2005;15(6):596-603.

35. Hanten WP, Schulthies SS. Exercise effect on electromyographic activity of the vastus medialis oblique and vastus lateralis muscles. Phys Ther. 1990;70(9).561-5.

Recebido: 05/10/2012

Received: 10/05/2012

Aprovado: 07/04/2013

Approved: 04/07/2013 